\section{Memoria del Presidente}

Santiago, Diciembre 16 de 2009

Apreciados Maestros y Ex-Presidentes de la Sociedad de Cirujanos de Chile, estimados socios y socias. Como es tradicional, me corresponde en esta sesión extraordinaria dar cuenta de la marcha de la Sociedad durante el año 2009, durante el cual tuve el privilegio de presidir los destinos de nuestra Institución.

\section{Sesiones Ordinarias}

Se cumplió con el $100 \%$ de las sesiones ordinarias programadas en el Auditorio de la Clínica Alemana, 3 de ellas con conexión a Provincia. Varios Departamentos de la Sociedad estuvieron representados en esta actividad que, a pesar de los incentivos que se han intentado en distintos directorios, reúne a un número reducido de socios y residentes de Cirugía, lo que no es representativo de una Sociedad que bordea los 900 socios. Probablemente el horario sea inadecuado para una actividad académica en el medio de la vorágine laboral y debemos explorar nuevas alternativas para mejorar la convocatoria de una actividad fundamental en toda Sociedad científica.

8 de abril:

- "Rol actual de la biopsia rápida".

Moderador: Dr. A. León.

\section{3 de Mayo:}

- "Utilidad del PET-CT en cirugía". Dr. H. Amaral.

- "Técnicas percutáneas y angiográficas de apoyo a la cirugía". Dr. P. Vargas.

10 de Junio:

- Homenaje al Dr. Jorge Kaplan K. Dr. L. Caimi

- "Aspectos diagnósticos y terapéuticos de la ictericia obstructiva”. Moderador: Dr. JC Díaz.

8 de Julio:

- "Urgencias de colon ¿qué hacer?" Moderador: Dr. R. Cárdenas.

12 de Agosto:

- "Prevención en trauma y manejo integral de víctimas". Moderador: Dr. L. Ristori.

09 de Septiembre:

- "Rol actual del cirujano en el tratamiento de la diabetes mellitus tipo II". Drs. I. Fernández y C. Ovalle.

\section{1 de Octubre:}

- Trabajo de Ingreso: “Aneurismas aorto-ilíacos. Experiencia personal”. Dr. Juan Bombín. Informante: Dr. F. Ibáñez.

- "Simpatectomia en hiperhidrosis". Moderador: Dr. R. Aparicio.

\section{Apoyo y Participación en Cursos y Congresos}

Una de las tareas primordiales de la Sociedad de Cirujanos de Chile es estimular la actividad científica en las provincias y dar apoyo a los Capítulos y Departamentos que la conforman. En esta oportunidad destaco:

1. Congreso IFSO Latinoamericano. 01 al 04 de Abril de 2009. Viña del Mar con la asistencia de 120 cirujanos extranjeros de Argentina, Perú, Bolivia, Brasil, República Dominicana, Colombia, Ecuador, Venezuela, Costa Rica, Panamá, Estados Unidos, España y Portugal. Este importante Congreso organizado por el Departamento de Cirugía Bariátrica y presidido por el Dr. Wiliam Awad, tuvo el soporte técnico y financiero de la Sociedad de Cirujanos de Chile con un saldo final favorable para nuestra Institución.

2. Departamento de Mama: Curso "Manejo actual del cáncer de mama", 18 al 20 de Junio en la Ciudad de Puerto Varas. Fue organizado por el Departamento de Mama que dirige la Dra. María Eugenia Bravo con recursos propios.

3. Departamento de Trauma: Por renuncia del Dr. Jaime Morán asume en junio la Dra. Ana María Pacheco la presidencia, a quien se agradece especialmente su encomiable actitud de velar por el bien superior. Organizan con gran éxito el Simposium FELAC durante el Congreso que habría requerido de un salón de mayor capacidad.

4. Departamento de Cirugía Vascular: Realizó 3 Jornadas en las Ciudades de Coyhaique, Antofagasta y Santiago con la asistencia de 150 personas e invitados extranjeros. Felicitaciones al Presidente Dr. Mertens y su directorio por esta prolífica actividad de difusión y docencia cuyo financiamiento fue conseguido a través de un sistema de aportes de las Industrias sin costo para la Sociedad.

5. Cirugía Bariátrica: Bajo la Presidencia del Dr. Cristián Ovalle destaca el Congreso IFSO La- 
tinoamericano ya comentado, las $1^{\mathrm{a}}$ Jornadas Multidisciplinarias en Puerto Varas y el tercer grupo de cirujanos que viaja a Isla de Pascua. Otro hito fue la incorporación de miembros no médicos en calidad de adherentes al Departamento.

6. Departamento de Hígado, Vías Biliares y Páncreas: Aparte de la sesión ordinaria ya señalada, efectuó en Septiembre un Curso de "Actualizaciones en Cirugía para médicos del Sur", en Valdivia (Director: A. Venturelli). Además el Curso Oficial que versó sobre "Algunos aspectos de la Cirugía Hepática", en el Hospital Clínico de la Universidad de Chile, con asistencia de 65 médicos, en Octubre. (Director: JC Díaz) y el Curso Oficial del Congreso de Coquimbo - La Serena. Presidente: Dr. Carlos Benavides.

7. Departamento de Cirugía Torácica: Bajo la presidencia del Dr. Rodrigo Aparicio se concretó una activa agenda científica que incluyó 6 reuniones itinerantes en los más prestigiados centros y clínicas de Santiago; Curso de Postgrado: "Cáncer pulmonar estadio I y II", con el Dr. R. Rami Porta como invitado de España y 2 cursos de Postgrado en el Congreso.

8. Departamento de Investigación y Evidencia (Dr. Carlos Manterola): Simposio de Cirugía basada en Evidencias realizado en el curso del XXVIII Congreso Brasilero de Cirugía, realizado en la ciudad de Sao Paulo entre el 26 y 30 de julio. Asistencia aproximada de 140 personas.

Simposio de Investigación en Cirugía realizado en el curso del $60^{\circ}$ Congreso Uruguayo de Cirugía, realizado en la localidad de Punta del Este entre el 18 y 21 de noviembre. Asistencia aproximada de 50 personas.

Curso de Investigación en Cirugía efectuado previo al LXXXII Congreso Chileno e Internacional de Cirugía, realizado en la ciudad de Coquimbo en el mes de noviembre. Asistencia aproximada de 25 personas.

9. Departamento de Hernias. Curso Anual 28-29 de Agosto en Puerto Varas. Curso Pre-Congreso: "Cirugía de urgencia y Técnicas quirúrgicas en hernias de la pared abdominal”. (Dr. A. Jirón).

10. Participación en Congreso de la FELAC 2009, llevado a cabo en Caracas, Venezuela desde el 11 al 14 de Julio. En esta oportunidad el Dr. Italo Braghetto, Presidente de la FELAC período 20072009, hizo entrega de la presidencia a Venezuela.

\section{Congreso ALACP 2009, 20-23 de Julio en}

Guayaquil, Ecuador con una delegación de 17 médicos chilenos, obteniendo dos de los tres premios otorgados al mejor trabajo libre y mejor póster.

\section{Actividades Capítulo IX Región}

Marzo - Abril. Curso práctico "Metodología para la investigación en cirugía". Impartido en 2 sesiones semanales por mes. Dirigido a cirujanos y becados. Directora del Curso. Dra. Viviana Pineda.

Mayo. Conferencia: "Origen y ámbito de la bioética". Enfermera Sra. Wally Oplinger.

Junio. Conferencia: "Cirugía endovascular de la aorta". Dr. Luis Burgos de Cea.

Julio. Conferencia: "Radioquimioterapia en cáncer de vesícula biliar”. Radioterapeuta Dr. Roque Conejeros.

Agosto. Conferencia: "Intervencionismo en Abdomen". Radiólogo intervencional Dr. Marcos Tapia.

Septiembre. Conferencia: "Incendio en el campo quirúrgico ¿Mito o Realidad?”. Anestesiólogo Dr. Oscar Cherres.

Octubre. Conferencia: "Cáncer de Pulmón Sobrevida a 5 años" Dr. Antonio Baquerizo.

Noviembre. Conferencia: "Cirugía oncoplástica de la mama". Dra. Viviana Pineda.

Toda esta actividad académica representa una de las principales tareas de la Sociedad de Cirujanos de Chile, que en el pasado ha sido el soporte de Congresos de gran relevancia como la ALACP de 1999, FELAC de 2007 y, en el futuro cercano, lo será del Congreso Panamericano de Trauma 2013.

\section{Ingreso de nuevos Socios Afiliados}

Uno de los objetivos primordiales de todos los directorios ha sido ampliar el grado de representatividad de la Sociedad, pues sabemos que no estamos en ella todos los que debiéramos estar. Dado el marco regulatorio de las especialidades médicas que rige en Chile es claro que debemos ser pro-activos y desarrollar nuevos mecanismos para encantar y atraer al seno de nuestra Sociedad a todos los cirujanos del país, especialmente a los jóvenes, quienes idealmente debieran ingresar como miembro afiliado apenas hayan recibido su título de especialista. En los viajes a distintas ciudades de Chile, tuvimos la oportunidad de escuchar los problemas de los cirujanos jóvenes que viven una realidad distinta, generalmente alejados de los grandes centros universitarios. A raíz de ello, enviamos una carta a muchos cirujanos del país invitándolos a integrarse a la Sociedad, remarcando las ventajas de pertenecer a nuestra Institución. La respuesta a esta convocatoria fue extraordinaria. Sin duda esta invitación cayó en un terreno fértil y por diversas razones, entre las cuales se incluye el proceso de certificación de 
especialistas que está llevando a cabo el MINSAL, los concursos en las distintas regiones del país y la progresiva judicialización de la práctica quirúrgica, este año se incorporaron 110 nuevos socios, demostrando la relevancia de la Sociedad de Cirujanos de Chile como referente de la Cirugía Chilena, hecho sin precedentes y que nos hace soñar con alcanzar una "masa crítica" que incluya a la gran mayoría de los cirujanos de nuestro país.

\section{La Sociedad y el proceso de Certificación de Especialistas}

Como parte de ASOCIMED, la Sociedad ha impulsado y seguirá apoyando la certificación transparente y profesional de las especialidades y sub-especialidades médicas, cuyo reglamento transitorio fue aprobado en el año 2007. Vemos con preocupación la incorporación de 2 nuevas formas de certificación, a saber, quienes mantengan un convenio vigente con FONASA para la atención en la MLE como especialistas y quienes se hayan desempeñado como tales a lo menos 5 años en establecimientos del Sistema Nacional de Servicios de Salud, certificado por el Director del Servicio de Salud correspondiente. En principio el MINSAL había aceptado la sugerencia de CONACEM de suprimir la palabra "especialista" y que en la página Web apareciera como "convenio vigente en FONASA" y "desempeño" en la especialidad respectiva, lo que finalmente no se cumplió y desde octubre de 2009 los colegas involucrados aparecen con el título de especialista. No obstante la forma de cumplir con el reglamento y que los criterios de certificación podrían ser muy heterogéneos y discrecionales de la autoridad local, la Sociedad de Cirujanos de Chile ha sido invitada en algunos Servicios del país a participar en los GTA (Grupo Técnico Asesor) locales, nombrando a destacados cirujanos como integrantes de estas comisiones que analizarán los antecedentes de los postulantes. Esto representa para la Sociedad una manera de "mirar el proceso desde dentro", aunque estamos conscientes de que otros colegas pueden considerarlo como una forma de avalar un proceso poco transparente.

De este modo, la Sociedad envió a un representante experto en la formación de un cirujano para integrar la Comisión de Cirugía General solicitada por el MINSAL, que ha trabajado arduamente durante estos 2 meses para establecer los estándares mínimos que deben cumplir los cirujanos que postulen a la certificación por esta vía teóricamente transitoria. Se han definido 5 áreas funcionales en las áreas diagnósticas, educación a la población para la prevención, manejo integral del paciente, manejo de la patología quirúrgica propiamente tal y finalmente desarrollo de la especialidad para mejorar los estándares de la calidad. Para ello se contempla las acciones, los conocimientos y las experiencias que deben rendir los postulantes. Quiero agradecer al Dr. Jaime Contreras su apreciable colaboración en esta tarea primordial que debe enfrentar la Sociedad.

\section{Revista Chilena de Cirugía}

El año 2009 se inició con la buena noticia de la aceptación de la Revista Chilena de Cirugía en el Science Citation Index Expanded (SCIE), con lo cual adquiere la categoría de publicación ISI. Esperamos que esto constituya un nuevo incentivo para los cirujanos chilenos, que se traduzca en más y mejores trabajos publicados en nuestra Revista.

Felicitaciones al editor Dr. Julio Yarmuch por este logro fundamental.

\section{Aranceles FONASA}

Durante el año tuvimos reuniones con el Regional Santiago del Colegio Médico para tratar el tema de los aranceles, con el objetivo de incorporar nuevas prestaciones en uso y eliminar las obsoletas. En el mes de noviembre, se hizo un consolidado que incorpora 1.000 nuevas prestaciones, un tercio de ellas del ámbito quirúrgico. A pesar de las previsibles dificultades que pondrá Hacienda para regularizar esta lamentable situación, la Sociedad cumplió con entregar un listado completo y detallado de las prestaciones por Especialidad. El Colegio Médico confía en sensibilizar a la Autoridad Sanitaria para conseguir este objetivo.

\section{El Congreso}

La planificación del Congreso se inició en el mes de julio de 2008 en un escenario complejo derivado de la crisis internacional. A pesar de lo anterior, el evento se pudo realizar en condiciones normales en el complejo Coquimbo-La Serena con la presencia de 19 invitados extranjeros y 39 invitados nacionales que dieron realce a un evento que reunió alrededor de 900 congresistas. Luego de una ceremonia inaugural que nos llevó a soñar con las galaxias y las supernovas, se presentó alrededor de 500 ponencias entre temas libres, vídeo y pósters cumpliéndose a cabalidad el Programa preparado por el Comité Planificador. El Comité Científico premió a los dos mejores Pósters del Congreso, cumpliendo con el doble objetivo de estimular esta actividad y permitir una discusión más acabada en las seccionales de Temas Libres. En el aspecto financiero, ya hemos escuchado la cuenta del tesorero y estamos satisfechos de haber logrado los objetivos planteados en un escenario difícil, debido especialmente a los altos costos actuales que implica una sede de grandes dimensiones. A pesar de la belleza y de la amplitud del lugar, debo reconocer ciertas dificultades del centro de eventos elegido para permitir el desarrollo de 7 
actividades académicas simultáneas más el necesario espacio para el equipo de Secretarias y la incorporación masiva de los Pósters, proceso que es difícil lograr en forma perfecta fuera de Santiago. Esperemos que junto al crecimiento del país se construyan nuevos y amplios centros de eventos que permitan el cumplimiento de las actividades societarias en forma cabal y sin restricciones. Quiero expresar mis agradecimientos al Comité Planificador central y local de la $4^{\mathrm{a}}$ Región, al Comité Científico y todos los Departamentos y Capítulos por su dedicación y creatividad en el desarrollo de este Congreso.

Luego de un período de 8 años en el Directorio que culmina con el nombramiento de Presidente de la Sociedad de Cirujanos de Chile hace un año, deseo expresar mis agradecimientos a los miembros de Directorio por su permanente apoyo y amistad durante este período. Un agradecimiento especial a Margarita Ferrer, nuestra Secretaria Ejecutiva por su dedicación, capacidad y organización que permitió al Directorio cumplir con las tareas fundamentales que nos propusimos al aceptar este alto honor. Algunos logros están a la vista pero quedan muchas tareas pendientes. Será tarea de los próximos Directorios enfrentar estos desafíos. En lo personal, ha sido una oportunidad y un privilegio que me ha hecho crecer como persona y como profesional. Muchas gracias.

Dr. Guillermo Bannura 